\title{
LA AMAZONÍA EN EL FUTURO DE LA AMÉRICA DEL SUR: identificación de los ejes estratégicos socioambientales para la cooperación Sur-Sur
}

\author{
AMAZONIA NO FUTURO DA AMÉRICA DO SUL: \\ identificação dos eixos estratégicos \\ socioambientais para a cooperação Sur-Sur
}

\author{
Carlos Antonio Martin Soria Dall’Orso*
}

\begin{abstract}
El articulo busca identificar las oportunidades de desarrollo de políticas públicas y proyectos en materia socioambiental que pavimenten la transición hacia la Economía Verde a partir de una propuesta que recoja las necesidades y oportunidades que brinda la Amazonia hacia un desarrollo diverso bajo en carbono y con sostenibilidad social, ambiental y económica. Se revisa la situación actual del modelo extractivista con limitado desarrollo del aparato legal y filosófico de los derechos humanos hacia un modelo que basado en la comprensión del paradigma de los derechos humanos ayude a construir relaciones más igualitarias, ofreciendo oportunidades para el desarrollo de políticas públicas que favorezcan la diversificación del modelo de desarrollo y la incorporación de proyectos bajos en carbono que apoyen a la sostenibilidad de la Amazonia y sus poblaciones. Se revisara los principales problemas legales e institucionales que afectan a las poblaciones indígenas y rurales a fin de dar solidez al modelo de un desarrollo diverso apoyándose en el desarrollo de capacidades de su población.

Palabras-clave: Amazonia. Economía verde. Sostenibilidad. Carbono. Poblaciones.
\end{abstract}

\section{LA AMAZONIA Y SUS PUEBLOS}

La Amazonia, uno de los mayores bosques del mundo, es el hogar de pueblos indígenas, mestizos y descendientes de inmigrantes europeos. Ésta es en gran parte una zona rural remota con los pueblos que viven en economías de subsistencia, pero también es una red de vibrantes y caóticos centros urbanos y pueblos que acopian recursos naturales a través de una economía mercantilista apoyada en capital más relaciones de compadrazgo que han estado suministrando recursos naturales para el mercado mundial desde tan temprano como el Siglo XVI. La exportación de caoba, caucho, quinina, la fauna, y muchos otros productos siempre ha influido en la economía de la región, con un ciclo de auge y caída. El sendero de los productos naturales que salen de la región sirve como un canal a través de la economía de subsistencia, la economía mercantilista, y

* Pontificia Universidade Católica do Perú. Universidad Nacional Agraria La Molina. Universidad San Ignacio de Loyola. Universidad Pedro Ruiz Gallo (INTE PUCP).

Av. Bolivar s/n Pueblo Libre, Lima 21, Perú. carlos.soria@ pucp.pe la economía de libre mercado. El destino de la Amazonía está muy relacionados con las demandas del mercado mundial y la falta de voluntad de los gobiernos nacionales a invertir los recursos del gobierno en una zona regida por las economías locales que suministran al mercado mundial a través de las redes de flujo de recursos naturales hacia la exportación.

\section{EL PROCESO DE ORGANIZACIÓN DEL MOVIMIENTO INDÍGENA AMA- ZÓNICO}

Unos 370 pueblos indígenas amazónicos, con una población estimada en 1,6 millones de personas viven en 2.200 territorios y han tenido un proceso singular en el lado andino que ha terminado influenciado a toda la región a través de la consolidación de una organización indígena regional como la Coordinadora de Organizaciones Indígenas de la Cuenca Amazónica (COICA).

En 1969, tres organizaciones de los pueblos indígenas nacieron en la Amazonía peru- 
ana. Después de 3 años de intensos esfuerzos, el pueblo amuesha de la Amazonía Peruana Central creó el Congreso de Comunidades Amuesha (Brysk, 1996, p. 40). En la Amazonía norte del Perú se crearon dos organizaciones de los pueblos indígenas por el pueblo aguaruna del rio Potro y el río Manseriche y por los Achuar de los ríos Huitoyacu, Manchari y Shintusi (Dandler et al, 1998, p. 12). Desde entonces, se han creado un gran número de organizaciones de pueblos indígenas amazónicos.

Diez años después nació la primera organización regional amazónica e interétnica: el Consejo Aguaruna y Huambisa. Ese mismo año, nació la primera organización nacional con la creación de la Coordinadora de las Comunidades Nativas de la Selva Peruana, que en 1980 cambió su nombre por el de Asociación Interétnica de Desarrollo de la Selva Peruana (AIDESEP). En 1984, AIDESEP lideró el proceso de creación de la única organización Pan -Amazónica que representa a los ocho países de la cuenca: la COICA. En 1987, una segunda organización nacional nació con la creación de la Confederación de Nacionalidades Amazónicas del Perú (CONAP). AIDESEP es activo en la defensa de la tierra, oponiéndose a la minería y la prospección de petróleo en sus territorios y en las áreas protegidas, y la organización de las bases para planificar e implementar la educación, la salud y proyectos de producción (Varese, 1996).

El reconocimiento de las tierras de los pueblos indígenas ha tenido diversos procesos históricos, legislativos e institucionales. En Brasil, tras el desalojo inicial y la reubicación de los pueblos se inició luego un proceso de reconocimiento y demarcación de tierras indígenas según la Constitución de 1988 que recogió parte de la herencia del trabajo de la Fundação Nacional do Índio (Funai) y avanzo en consolidar el enfoque de derechos humanos, la diversidad de pueblos indígenas y procesos históricos ha generado una diversidad de territorios indígenas donde los más grandes fueron otorgados a los pueblos indígenas como en el caso de los yanomami.

El caso grave más reciente de criminalización de la protesta social en la Amazonia ha sido el levantamiento indígena y la masacre de Bagua. Durante 2007 y 2008, el gobierno de García (2006-2011) en el Perú buscó promover la inversión en tierras amazónicas para los biocombustibles, la soja y la madera. Estas intenciones causaron gran preocupación entre los pueblos indígenas y los pueblos no indígenas de la región amazónica peruana que se declararon en huelga exigiendo la anulación de esta legislación en 2008 y 2009. Después de muchos días de revuelta, el gobierno de García anuló dos decretos que favorecían de inversionistas a la parcelación de las tierras de los pueblos indígenas.

Si bien Bagua es quizás el caso más grave y conocido, éste es solo un ejemplo de un proceso de superposición de la sociedad nacional expandiéndose sobre áreas predominantemente caracterizados por derechos públicos y comunales donde estos últimos son obviados y ultrajados por los funcionarios en favor de los derechos de los inversionistas privados y a veces entre estos en favor de los más grandes. Estas protestas se dan en todo el continente en la medida que la inversión expande su presencia y sus categorías jurídicas (propiedad privada, concesión, imperio de la ley) para querer reorganizar paisajes de propiedad que son dinámicos (Coward, 2006) y carecen de liquidez por paisajes que entran a la dinámica de la propiedad privada, la inversión, la rentabilidad e introducen a las jornada laboral de 4048 horas.

En el caso de Colombia, las poblaciones rurales se encuentran en medio de la violencia que ha afectado a este país. Los pueblos indígenas son un blanco fácil para cualquiera de las fuerzas contendientes involucrados. Protestas similares ocurrieron en Colombia en 2008, donde los pueblos indígenas realizaron una marcha nacional a la capital del país, Bogotá, para presionar al gobierno por los derechos de la tierra y para denunciar la violencia. Hasta 1979, los pueblos indígenas no 
votaban en Ecuador, fue entonces que la obligación de ser alfabetizado fue eliminada y pudieron votar y ser elegidos (Mumme; Korzetz, 1997, p. 49). Mumme y Korzetz señalan que los indígenas de Ecuador han defendido sus intereses ambientales a través de la representación ofrecida por CONAIE y no a través de "representación basada electoralmente” (p. 49). El proceso de organización se expandió gradualmente en toda la región amazónica ecuatoriana. En 1992, 2.000 indios amazónicos marcharon hacia Quito y exigieron derechos sobre la tierra; como resultado, el saliente presidente Rodrigo Borja (1988-1992) otorgó derechos a la tierra en una medida que superó los títulos de las tierras dadas por todos los gobiernos anteriores juntos (Red de Acción de Bosques Tropicales, 1997; Sawyer, 1997). Los pueblos indígenas amazónicos y sus organizaciones han sido actores claves de los movimientos sociales y de los levantamientos que depusieron a los presidentes Bucaram, Mahuad y Gutiérrez. Mientras el gobierno de Correa tuvo un inicial apoyo de los indígenas para luego enfrentar sus demandas de reforma agraria sobre la tierra y la salida de Correa para evitar su tercera elección.

En Bolivia de manera similar el inicial apoyo de los indígenas amazónicos pronto se volvió en confrontación y repudio por un gobierno más proclive a atender las demandas de su electorado cocalero y colono.

Todos los gobiernos nacionales amazónicos declaran respeto de los derechos de los pueblos indígenas y los compromisos de la legislación internacional de protección de sus derechos, mientras que las autoridades regionales y locales quieren evitar la implementación más mínima de estos derechos. Los programas financiados por el Banco Mundial y otras instituciones han favorecido la titulación individual de la tierra para los colonos pero han demorado en favorecer la titulación de tierras comunales de los pueblos indígenas.

\section{LOS PUEBLOS INDÍGENAS EN AIS- LAMIENTO Y CONTACTO INICIAL ${ }^{1}$}

Brasil ha liderado el desarrollo de la política de demarcación y protección de los territorios de los pueblos indígenas en aislamiento y contacto inicial, así como los arreglos institucionales para ello. Aunque no todos los esfuerzos tuvieron éxito, estos contribuyeron al proceso de comprensión de la necesidad de no forzar el contacto con estos pueblos y de respetar el derecho a sus tierras. Desde la creación de FUNAI 13 millones de hectáreas de bosque amazónico se han reservado como áreas para los pueblos indígenas en aislamiento. En Brasil, hay una estimación de alrededor de 40 pueblos indígenas en aislamiento que se han verificado y además de 27 no verificados, que representan un total de 67 pueblos indígenas en aislamiento.

En el caso de Perú se estima que hay 20 pueblos en aislamiento y 14 en contacto inicial con 5 reservas territoriales ${ }^{2}$ sobre más de 2’812,000 hectáreas. Sin embargo, otros cinco propuestas de reservas territoriales ${ }^{3}$ han estado esperando la aprobación del gobierno, algunas de ellas desde 1999.

En Bolivia, en 2006, el gobierno creó la Zona Intangible de protección integral y absoluta reserva de los pueblos Toromona en el Parque Nacional Madidi, que también está habitada por el pueblo Ese Ejja y los pueblos

${ }^{1}$ Unos 85 pueblos indígenas en aislamiento viven en los bosques de los países amazónicos. Hay 67 de ellos en Brasil, 14 en Perú y uno de ellos, al menos, en Bolivia, Colombia y Ecuador. Los pueblos indígenas en aislamiento habitan cabeceras, colinas y otros terrenos altos a su disposición. Muchos permanecen en los bosques donde la fauna es más abundante. Viven de la caza, pesca, recolección y agricultura incipiente. Los informes coinciden en su migración estacional a lo largo de las riberas para recoger huevos de tortuga. En estas ocasiones se mueven en grupos familiares de alrededor de 25 personas, estableciendo campamento cada cinco kilómetros después de caminar un día.

2 Estas son la Murunahua y la Mashco Piro en la región Ucayali (1997), la Isconahua en la región Ucayali (1998), la Madre de Dios para los pueblos Mashco Piro (2002) y la Kugapakori Nahua Nanti en las regiones Ucayali y Cuzco (2003).

${ }^{3}$ Estos son las propuestas en el Yavarí Tapiche, Yavarí Mirim, Napo Tigre Curaray en Loreto, la Kapanawa en Loreto y Ucayali, y la Cacataibo en Loreto y Ucayali. 
Kapuibo. Estos indígenas viven en un parque nacional, un área de protección estricta con la prohibición de uso de los recursos por parte de terceros.

Esta figura se repite en el Parque Nacional Rio Puré en Colombia y en la Reserva Territorial Mashco Piro dentro del Parque Nacional Alto Purús. Igualmente en Ecuador, en 1999, el gobierno ecuatoriano creó la Zona Intangible Tagaeri Taromenani para 'proteger la vida e integridad de los Tagaeri Taromenani', dentro de una Area Nacional Protegida (ANP) de carácter estricto pero rodeada por actividades extractivas.

En 2002, el gobierno colombiano estableció sobre 999.880 hectáreas el Parque Nacional Natural del Río Puré para proteger a los pueblos Aroje, Yuri o Caraballo, respetando su derecho a permanecer en aislamiento. Esta categoría permite a los pueblos establecerse en el futuro convirtiendo el parque nacional en tierra comunal titulada a los pueblos indígenas.

El trabajo forzoso sigue siendo una situación común en Bolivia y Perú, tanto en la minería de oro artesanal y en la extracción forestal. Trabajadores andinos son contratados en sus pueblos y enviados a los bosques y los ríos amazónicos para extraer los recursos. Ello ocurre en condiciones laborales del siglo XV y mientras usan el equipo chino barato siglo XXI para buscar oro en los lechos de los ríos amazónicos. Un pequeño inversor por lo general tiene . un permiso de explotación que no es superviผे sado in situ. Un trabajador es contratado por un período de dos años de trabajar seis días a la semana, sin días festivos y una cuenta de crédito que puede extender su contrato.

Estos pequeños centros urbanos son ciudades que se encuentran a la vanguardia de la expansión de la frontera y sirven como nodos en las redes de flujo de los recursos naturales que fluyen hacia los mercados regionales, nacionales e internacionales. Así, por ejemplo, los grandes bagres de los ríos Ucayali y Marañón y sus tributarios como el Pacaya y el Samiria, pescados relativamente cerca de los asenta- mientos ribereños, salen en cajas de madera con hielo seco hacia Leticia y de ahí al resto del mercado nacional colombiano. Asimismo las nueces de Brasil y otros productos como cueros de diversos animales amazónicos también tienen sus propias redes de comercialización desde el bosque hacía en el mercado mundial.

Fuera de la mayoría de estas ciudades y los pueblos amazónicos más pequeños, la Amazonia sigue siendo un paisaje rural. $\mathrm{Mu}-$ chas praderas y pastos dominan áreas de los ríos amazónicos del este, mientras que en la Amazonía brasileña, la expansión de una red de carreteras iniciada en 1950 dio lugar a cultivos modernos de soya que dominan el paisaje. En la Amazonía andina, un área colonizada en muchos lugares desde hace 150 años, los descendientes de los colonos europeos han obligado a los pueblos indígenas a los márgenes de los valles si es que lograron subsistir en un paisaje ahora ocupado por pastos, praderas y asentamientos urbanos de estilo europeos. Más allá de estas áreas las economías de subsistencia de los pequeños colonos y los pueblos indígenas ocupan los afluentes más pequeños con sus asentamientos familiares. En la Amazonía occidental, muchos pueblos indígenas que viven en la parte superior de los afluentes conservan sus trajes tradicionales, el lenguaje y la organización, mientras que los pueblos indígenas de las tierras bajas son, sociedades ribereñas abiertas al comercio desde tiempos muy antiguos. $^{4}$

Por lo general toma 30 años de adaptación al cambio y experiencia propia para que muchos recién llegados puedan comprender que la producción agrícola en las tierras tropicales es muy diferente a la agricultura de las tierras de regadío. En algunos casos, la población migrante de una zona rural ha estado allí durante bastante tiempo de haber integrado el conocimiento tradicional con la explotación de su ambiente amazónico. Después de una

${ }^{4}$ El comercio tradicional indígena amazónico involucraba hojas de coca, sal, cuchillos y hachas piedras, algodón y fauna, entre otros productos. 
o más generaciones, esta mezcla ha producido una cultura amazónica criolla de 'pueblos ribereños’ y extractores (ribereños en español, ribeirinhos en portugués) - descendientes de migrantes que durante generaciones se habían adaptado a la utilización de los pueblos indígenas de recursos- que son en parte una mezcla de creencias que implican conocimientos tradicionales sobre cómo cosechar el ambiente. Mientras que Brasil ha otorgado derechos a la tierra a estas poblaciones, otros países como Perú no han reconocido derechos a la tierra de estos pueblos 'migrantes' y sus descendientes, a pesar del hecho de que muchos de ellos viven en el bosque y se alimentan de ella. Perú recién con su nueva ley forestal 29.765 de 2011 reconoce derechos de uso del bosque a poblaciones rurales diferentes a los indígenas.

\section{EL COMERCIO FORESTAL}

A partir de 1990 algunos esfuerzos por producir una reforma en el bosque tropical brotaron en los países amazónicos. Bolivia, Brasil y Perú aprobaron nuevas leyes para alejarse de la extracción sin gestión a fin de promover la gestión sostenible de los bosques. Se promovió sistemas de certificación forestal y el apoyo de la Agencia de los Estados Unidos de América para el Desarrollo Internacional (USAID) ayudó a Bolivia experimentar con la reforma de los bosques con su proyecto BOLFOR. Perú hubo de esperar hasta 2002 para comenzar a otorgar concesiones forestales también con el apoyo técnico de USAID. En Perú, entre 2002 y 2004, se otorgaron concesiones forestales sobre aproximadamente 7,8 millones de hectáreas. Dichos concesionarios usaron la política para evitar pagar los precios ofrecidos por el derecho de aprovechamiento en muchos casos. Muchas concesiones fueron devueltas y solo algunas lograron manejar el negocio debido en mi opinión a condiciones de escala del negocio, potencial de la concesión y de condiciones organizacionales de las empresas que las operan.

Sin embargo, la corrupción, la mala gestión y las políticas de corto plazo a favor de la reforma no cambiaron la relevancia y la prioridad de la tala ilegal en las políticas públicas del sector forestal. Aunque las concesiones forestales son la fuente principal oficial de la producción de madera, los permisos a los productores agrícolas para la conversión del hábitat también les permiten vender la madera, mientras que otros millones de hectáreas de tierras forestales están en manos de pueblos indígenas; quienes son buscados cada vez más por los madereros con el objetivo de establecer contratos de acceso a la madera. Las áreas protegidas y reservas territoriales para pueblos indígenas en aislamiento son también una importante fuente de madera ilegal.

Un aspecto curioso es que desde 2004 hasta 2010, Fiji exportó 6 veces más caoba que Bolivia, Brasil y Perú juntos (Rosales, 2013, p. 62-215). Por la década de 1960, el gobierno de Fiji comenzó a plantar plántulas de caoba en gran escala. Para la década de 1990, las plantaciones habían crecido sobre más de 32,374 hectáreas. En 2000 hubo agitación política en Fiji por el interés de explotar estos recursos, incluyendo un golpe de estado y la confrontación de los intereses británicos y estadounidenses sobre la explotación de la caoba de Fiji (Frank, 2000; Soria 2015a).

Los pueblos indígenas son los más profundamente afectados por el comercio de la madera que drena sus bosques y muchas veces también la confianza en sus organizaciones o autoridades. Los pueblos indígenas se ven afectados por la reforma forestal a través de la superposición de concesiones forestales con tierras indígenas, en particular, la tierra que aún no está registrada; la falta de un adecuado proceso de consulta previa a la adjudicación de todas las categorías de manejo forestal; la invasión de las tierras comunales y las reservas territoriales para pueblos indígenas en aislamiento; además del fracaso de los sucesivos gobiernos para cumplir con sus ofer- 
tas de asesoramiento técnico en apoyo de la gestión forestal comunitaria hasta la reciente aprobación de la nueva ley que ofrece nuevas esperanzas a este viejo pedido de los indígenas y otros pobladores del bosque. Al mismo tiempo, estos pueblos indígenas suelen verse afectados por la corrupción en el mercado de la tala ilegal.

Los recientes avances en la descentralización no ofrecen mucha esperanza para los bosques. Los políticos regionales amazónicos suelen ser orgullosos representantes de los intereses de la madera ilegal. Por ejemplo, el presidente regional de Loreto, anunció en 2008 en una conferencia internacional que asistió el Ministro de Medio Ambiente de Brasil, que Loreto no otorgaría un metro cuadrado más de áreas protegidas o territorios indígenas, de conformidad con las exigencias de los madereros y otras industrias extractivas. El liderazgo político regional está muy influenciado por las fuerzas económicas regionales, como la madera y el oro ilegal que en muchos casos son también fuertes actores políticos locales.

\section{MEGAPROYECTOS}

La asimetría entre los pueblos indígenas, otras poblaciones rurales, inversionistas forestales y actores involucrados en el uso del y las industrias extractivas es un tema central ํ. en los conflictos socioambientales que ocurஸे $\rightarrow$ pavimentación de los ya existentes fomentan s la llegada de nuevas poblaciones buscando la 될 tierra y sus recursos; lo que da lugar a la expula sión de la antigua población sin títulos de proiิ piedad o capital a nuevas áreas de deforesta-

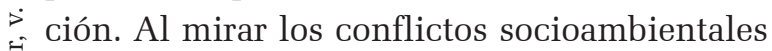
en torno a políticas de recursos naturales, nos encontramos con un divorcio entre el discurso de la legislación y de los funcionarios públicos y la puesta en práctica del mismo en todos los niveles. Los supuestos del marco legal tienen muy poca relevancia para los usuarios de recursos que toman decisiones en los bosques sobre la forma de gestionar los recursos, la forma de resolver los conflictos y la forma de compensar daños y perjuicios. En estas circunstancias, el nuevo otorgamiento de concesiones forestales o la apertura de nuevas zonas por la Iniciativa de Integración Regional Sudamericana (IIRSA por sus siglas en español) sugiere la intensificación de los conflictos que afectan a los pueblos, colonos, campesinos y poblaciones ribereñas indígenas debido al aumento en la demanda de tierra, los bosques, minerales, petróleo y gas, entre otros. Estos procesos han sido graficados por la red RAISG en sus mapas de las presiones que afectan a la Amazonia y sus poblaciones. ${ }^{5}$

IIRSA es una propuesta para construir no sólo los caminos que salen de Brasil para conectarse con sus vecinos, sino también a las carreteras entre los vecinos de Brasil, así como los puertos en el Amazonas y en ambos océanos y las redes de comunicación. En Perú, IIRSA construyó la Interoceánica Norte (Paita-Yurimaguas-Huallaga-Río Amazonas) y la Interoceánica Sur (Ilo-Cusco / Puno-Madre de Dios-Assis). Mientras que se ha propuesto una Interoceánica Central desde (Lima) Pucallpa a Cruzeiro do Sul. El diseño propuesto atravesaría una propuesta de área protegida en Sierra del Divisor Nacional y la Reserva Territorial Isconahua para pueblos indígenas en aislamiento en Perú; así como al Parque Nacional Serra do Divisor en Brasil. Hoy sabemos que antes que el desarrollo ha estado el deseo de sembrar concreto para generar jugosas comisiones que comienzan a ser investigadas en Brasil.

Sin embargo, muchos países amazónicos carecen de arreglos institucionales adecuados para la gestión ambiental y social adecuada del desarrollo de infraestructura. Algunas de las instituciones financieras internacionales, sobre todo del sector privado, también carecen

${ }^{5}$ Amazonía bajo presión presenta mapas de las presiones actuales y las amenazas potenciales(carreteras, petróleo y gas, minería, hidroeléctricas, focos de calor y deforestación) para toda la Amazonía. Vea la informacion disponible en http://raisg.socioambiental.org/amazonia-bajo-presion-2012 
de estas normas adecuadas. Algunos gobiernos ven estas normas como un obstáculo para el desarrollo de las inversiones y prefieren, evitar la introducción de las reformas necesarias o debilitar los mecanismos ya existentes.

\section{PETRÓLEO Y GAS}

En el caso de los efectos ambientales y sociales del desarrollo de la energía mis estudios en Ecuador y Perú a lo largo de la década de 1990 (Soria, 2014) mostraron cómo el proceso de desarrollo del marco jurídico pone de manifiesto cierta tensión entre el texto de la ley y el cumplimiento del hecho social (Habermas, 1997, p. 1-9). Por un lado, está el texto normativo, la letra de la ley, la expresión de los resultados del debate político y, paralela a ella, corre la interpretación social de los hechos, la interpretación hecha por los políticos, el sector privado, las organizaciones no gubernamentales y el público en general. En el caso ecuatoriano mostré la tensión entre la legislación que prohibía la contaminación por hidrocarburos en las áreas protegidas y la interpretación final hecha por el presidente Durán Ballén (19921996) que permitió las actividades petroleras en la Reserva de Producción Faunística Cuyabeno. Esta fue la definición de una serie de diversas interpretaciones de la ley sobre la operación de empresas petroleras en 'áreas protegidas' de la Amazonía ecuatoriana. En el caso peruano, esta tensión dio lugar a la derogación de una tercera parte del Código del Medio Ambiente de 1990 con el fin de favorecer la inversión extranjera, hecho que ocurrió entre 1991 y 1992. Sin embargo, a principios de 1999, el gobierno peruano aprobó la Estrategia Nacional de Áreas Naturales Protegidas por el Decreto Supremo 10-99-AG. Este decreto establecía que los recursos no renovables podrían ser explotadas sólo en áreas protegidas en las que se permite el uso directo de los recursos, una política que todavía está en vigor hoy.

Desde 2004, un segundo boom del pe- tróleo y el gas alimentado por las crecientes economías de Estados Unidos, Europa, China y la India y un alza de precios que alcanzó los US \$140 por barril en el 2008 expandió la actividad petrolera en el Perú de un 13\% del territorio de la Amazonía peruana a un $75 \%$ de esta región, que ocurrió en medio de una deficiente aplicación de la gestión ambiental y la falta de recursos para operar en un nivel adecuado, manteniendo normas legales muy laxas. El ejemplo más claro fue el gasoducto Camisea que se rompió cinco veces en su primer año de funcionamiento. La presión política vino directamente del Presidente Alejandro Toledo, quien declaró que el gas tenía que llegar a Lima el 9 de agosto de 2004, resumió la actitud en la frase 'Camisea, como sea'.

En Brasil las actividades petroleras se produjeron principalmente en la costa. En la década de 1990, un derrame de petróleo en Rio de Janeiro obligó a un cambio en apoyo de mejores políticas ambientales en Petrobras. Más tarde, cuando Petrobras quiso entrar en los campos de petróleo de Urucu tuvo que mejorar sus estándares ambientales y sociales. Aun así fue necesaria la demanda organizada de las poblaciones locales a lo largo del gasoducto Urucu - Manaos para obtener algo del gas con un plan de gestión ambiental de US\$ 20 millones y un plan social de US \$ 20 millones, equivalente al $2.66 \%$ de la inversión total de 1500 millones. Mientras en 6 lotes revisado en Perú la gestión social y ambiental de las inversiones de hidrocarburos bordeaban el $0.33 \%$ de la inversión (Soria, 2015f).

\section{LA NECESIDAD DE CAMBIOS EN LA GESTIÓN LAS POLÍTICAS PÚBLI- CAS SOCIOAMBIENTALES}

El desarrollo planificado solo por la mano de los intereses individuales no aborda las cuestiones más amplias de la resiliencia, la sostenibilidad y la gobernabilidad. Estas últimas cuestiones son consideraciones centrales 
en la planificación de un futuro sostenible para la Amazonía, sobre todo desde una mirada colectiva, regional y global. Algunos gobiernos regionales y locales de la Amazonía, la sociedad civil y los movimientos sociales están exigiendo la rendición de cuentas en el proceso de asignación del uso del suelo. Mientras tanto la superposición de usos múltiples es la tradición de los estados. Sin embargo, cuando uno de estos usos restringe la posibilidad de los otros usos, como en el caso de industrias extractivas, la invitación al conflicto social está lista, como los procesos de malestar social vividos en Bolivia, Brasil, Colombia, Ecuador y Perú lo demuestran.

La propuesta de economía verde desea mantener los procesos ecológicos esenciales, conservar la diversidad biológica y de ecosistemas, la estabilidad del clima en el marco del crecimiento económico sostenible y la mejora de calidad de vida de las poblaciones.

Los procesos de pago por servicios ambientales y Reducción de Emisiones por Deforestación y Degradación (REDD) están siendo implementados por el Banco Mundial y el Programa de Naciones Unidas para el Desarrollo promoviendo cambios legales, institucionales, investigaciones y programas de conservación de bosques en países priorizados como Perú, Congo, Indonesia y otros. El financiamiento REDD trae fondos frescos para conservar los ๑ bosques a través de promover dialogo, acuerN dos de conservación, concertación de políticas की de buen gobierno y de excelencia ambiental, con respaldo de la población local.

ริ Brasil, Colombia y Perú se encuentran 될 entre los primeros países en iniciar los arre\& glos legales e institucionales para REDD. Esta 今 es una gran oportunidad, si consideramos los millones de hectáreas de bosques de diversos tipos que se pueden beneficiar de recursos financieros y técnicos para su conservación y manejo sostenible. Ello se refleja, por ejemplo, en la existencia de más de 30 proyectos REDD en las diferentes regiones del Perú, incluyendo dos regiones priorizadas como San Martin y
Madre de Dios. En 2013, el gobierno y los pueblos indígenas de Perú acordaron implementar proyectos REDD + y comenzaron a trabajar en la arquitectura jurídica e institucional para que sea posible. En noviembre de 2013, durante la Segunda Cumbre de la COICA, COICA acordó con el Ministerio del Ambiente (MINAM) de Perú avanzar hacia la COP 20 dando apoyo a la propuesta de la REDD Indígena Amazónico con el objetivo de asegurar la tenencia de la tierra, los bosques y los recursos y promover acciones de conservación para mantener los bosques en pie y la prestación de servicios ambientales.

Desde 2011 las organizaciones indígenas han dialogado con el Estado peruano en el marco de la elaboración participativa de la Ley Forestal y de Fauna Silvestre y su reglamento que recoge el aporte de los pueblos indígenas en la norma, incorpora el conocimiento tradicional, la cosmovisión indígena y da mecanismos para la autonomía en el manejo de subsistencia de sus recursos naturales, así como la posibilidad fortalecer su participación en la administración forestal. En 2015 concluyó el proceso de consulta previa del reglamento general y sus seis reglamentos específicos. De manera que pueblos indígenas y Estado han estado caminando hacia el encuentro y el planteamiento de un futuro común para la humanidad que es salvar los bosques y adaptarnos al cambio climático.

En este marco las organizaciones de pueblos indígenas CONAP y AIDESEP han venido acordando con el gobierno peruano pero también con ONU REDD, Banco Mundial y otros, un marco general para la implementación de proyectos REDD. La Conferencia de las Partes 21 de la Convención Marco de Naciones Unidas sobre Cambio Climático en 2015 propuso los esquemas y la arquitectura institucional y operativa definida para implementar esquemas REDD a nivel internacional de manera de alcanzar los nuevos límites de GEI y atraer inversiones REDD, las mismas que en 2014 ya eran más de 400 millones de dólares solo para pueblos indígenas.

Los grandes desafíos hacia delante tie- 
nen que ver con construir la transición hacia un paradigma basado en la sostenibilidad del aprovechamiento del ambiente; para lo cual se requiere un profundo cambio de valores, de reglas y de conductas. La política y la educación pueden hacer posible el cambio de paradigma económico hacia la sostenibilidad.

Este cambio ya ha iniciado por ejemplo en el Perú a través de la reciente Ley de Servicios Ecosistémicos (REDD y biodiversidad); la elaboración participativa del Proyecto de Reglamento de la Ley Forestal y de Fauna Silvestre y su aprobación en consulta con los pueblos indígenas amazónicos. Sin embargo aún hay desafíos para construir una economía verde en el mundo rural y resolver las incongruencias en el marco legal de tenencia de la tierra, o de la seguridad jurídica para todas las formas de tenencia de la tierra (pública, privada y comunal).

Se espera que estos y otros cambios permitan superar los desafíos institucionales como contar con un mecanismo judicial rápido de atención de quejas sobre afectación de tenencia, delitos contra el ambiente y otros; la definición de competencias claras integradas en un sistema de los múltiples involucrados (Ministerio de Agricultura, Ministerio del Ambiente, Gobiernos Regionales [subnacionales] y Otros); la implementación de una institucionalidad estatal efectiva en el medio rural; la integración de políticas de conservación con políticas de crecimiento económico en los tres niveles del Estado.

\section{CONCLUSIONES}

De manera que es evidente en los países de la Amazonia el inicio de la transición para el cambio de paradigma de la extracción hacia la sostenibilidad. En Perú la nueva Ley Forestal y de Fauna Silvestre con enfoque intercultural y respeto a derechos de pueblos indígenas, así como su posterior proceso de consulta previa con los pueblos indígenas son señal de un avance regional importante que puede expandirse por la región, donde varios experimentos en esa dirección se han venido dando a nivel administrativo pero no legislativo.

De manera que hay oportunidades para el desarrollo de un marco legal adecuado a la realidad diversa de la Amazonia y sus pueblos indígenas, desde los indígenas en aislamiento hasta las comunidades nativas con certificación forestal voluntarias. Hay excelentes oportunidades en materia de REDD y manejo forestal como lo demuestran los avances entre el Estado y los pueblos indígenas en ambas materias. Sin embargo se ha hecho muy poco en la identificación de las medidas y las estrategias de adaptación y mitigación al cambio climático en las comunidades nativas y campesinas. Los proyectos REDD que están comenzando a implementarse son una oportunidad para ello. Adicionalmente hay la necesidad de desarrollar los mecanismos de salvaguardas de los derechos de los múltiples involucrados en los proyectos REDD lo que es una oportunidad para mejorar la gobernanza del bosque. Hay grandes oportunidades para pavimentar el camino hacia la Economía Verde a partir del impulso de la OECD, PNUD y BM. La COP 21 fue una oportunidad clave para consolidar las propuestas REDD+ como RIA así como para visibilizar el potencial de desarrollo futuro que ofrecen los bosques.

Es necesario desarrollar investigación a nivel de los modelos conceptuales por un lado, mientras que de otro lado, es necesario identificar dentro del territorio nacional las áreas del país donde podría implementarse experiencias piloto de Economía Verde. Como nos ha dicho Orstrom (1990) los bienes comunes como los bosques, los ríos y la atmosfera requieren ciertas características para permitir su manejo, así como Djeumo (2001) también señala que también es importante entender las barreras que tiene que superar el grupo social que maneja el recurso. En mi opinión este criterio ha sido clave también en el caso de las concesiones forestales amazónicas. 
La política ambiental recién, con el proceso de elaboración del RLFFS está iniciando una refundación de los meta valores de las normas desde una perspectiva ecosistemica e intercultural que nos permitirá darle contenido a una Economía Verde desde el Sur.

\section{Sobre la economía verde y las políticas para la sostenibilidad}

Con el fin de controlar realmente el bienestar de los bosques se requiere no solo los datos detallados sobre cada envío por especie, volumen y demás sino que es necesario que haya una adecuada gobernanza en los bosques que favorezca el manejo forestal sostenible. En ese sentido las experiencias de planes de manejo y acuerdos de conservación y gestión de recursos naturales.

Proponer a las plataformas internacionales como CITES CBD y UNFCC nuevas medidas de análisis integrado de los objetivos de estos tratados y los mandatos a sus conferencias y sus miembros para garantizar el bienestar de los bosques, asegurando la tenencia legal de los mismos en sus varias formas de propiedad y el manejo forestal sostenible.

Los países amazónicos en el marco de la Convención Marco de Cambio Climático están definiendo como colaboraran para mitigarlo y adaptarse. Para ello se definen contribuciones จ nacionales y se definen planes de acción en กิ estos temas.

Un factor clave es el ordenamiento y la generación de información que de sustento cualitativo y cuantitativo a este nuevo discurso de meta valores que requieren ser medidos, of gestionados y monitoreados a fin de que las acciones produzcan resultados meritorios.

La nueva generación de micro satélites multi-espectrales de hasta 500 bandas ofrecen un instrumento de análisis y modelamiento de escenarios más accesible a los centros de investigación y ONGs, asimismo la tecnología Drone está permitiendo a las organizaciones indígenas fortalecer su monitoreo del territorio.

La economía verde y los mercados de carbono así como las demandas de la Organización para la Cooperación y el Desarrollo OCDE por un presupuesto basado en resultados requieren de monitoreo, reporte y verificación de las acciones y resultados alcanzados. En materia de tenencia de la tierra ello supone acciones abiertas en la promoción del ordenamiento, el manejo y el reporte pero también acciones encubiertas y coordinadas de combate a las actividades ilegales que operan a través de medios informales y afectan a tierras y poblaciones vulnerables.

Es necesaria la gestión del conocimiento a través del desarrollo de capacidades instaladas en los varios niveles del estado y la sociedad civil; el desarrollo de redes de conocimiento y la promoción de espacios de reflexión investigación sobre la problemática socioecosistemica.

Es necesario mejorar la relación entre ciencia y política, tanto comunicando ambos espacios como generando mensajes adecuados para dicha comunicación; esto es desarrollando estrategias de compartir resultados de investigación que ayuden a retroalimentar la gestión de las políticas públicas en sus varios niveles.

\section{Sobre los desafíos socioambientales para la Sostenibilidad}

Los países amazónicos han venido desarrollando su política de protección de reservas indígenas con una legislación que suma un centenar y medio de normas de varios niveles sobre todo en 5 de los países amazónicos, los mismos que han avanzado en desarrollar Reservas para pueblos indígenas en aislamiento y contacto inicial (Véase Soria 2015b; d and e).

Los estados están decidiendo el papel de los territorios de los pueblos indígenas en aislamiento y contacto inicial en relación con proyectos REDD. En el caso de Perú estos fon- 
dos son para estos pueblos y deberían destinarse a su bienestar y el de sus territorios a través del Ministerio de Cultura y las organizaciones indígenas. En el caso de Brasil el Estado percibe los beneficios de estos territorios.

Los proyectos REDD son una oportunidad de transición hacia la economía verde y también hacia la consulta previa libre e informada en la medida que se use como instrumento para asegurar la salvaguarda de los derechos de los pueblos indígenas y el bienestar de la sociedad y el ambiente. En el corto plazo, la necesidad del consentimiento fundamentado previo de los pueblos indígenas parece ser la clave para el desarrollo de las políticas públicas de los pueblos indígenas, como lo muestra el proceso peruano a pesar de sus dificultades.

A largo plazo, el proceso de urbanización y su tasa de crecimiento sugieren que el desarrollo no planificado continuará agotando el bosque, lo que afectará el clima regional a través de la reducción de la precipitación de agua y el aumento de la temperatura. Mientras avanzamos hacia la COP 2030, el mundo busca que los mercados ayuden a salvar el clima a través de financiar la reducción de emisiones de gases de efecto invernadero (GEI) que generan la deforestación y la degradación de los bosques.

\section{Sobre las oportunidades de Cooperación Sur Sur}

A nivel de la Organización del Tratado de Cooperación Amazónica (OTCA) se ha venido avanzando promoviendo el dialogo intrarregional alrededor de temas como pueblos indígenas o gestión integrada de recursos hídricos entre otros, sin embargo estos espacios internacionales se hacen realidad a través de la coordinación de las cancillerías respectivas, las que generalmente no conocen mucho detalle del tema técnico y difícilmente adoptan medidas más allá de declaraciones generales de interés sin financiamiento ni mandato legal mínimo.
Algunas instituciones han avanzado en el dialogo interinstitucional como FUNAI de Brasil y MININTER de Colombia, Ministerio de Justicia de Ecuador, o MINCUL de Perú, quienes han venido trabajando temas comunes en la política publica de protección de pueblos indígenas en aislamiento y contacto inicial. Perú ha desarrollado legislación en el tema mientras Brasil ha desarrollado una práctica de varias décadas de acción pública en apoyo a estos pueblos. Bolivia, Ecuador y Colombia tienen mucho que beneficiarse en un esquema de cooperación amazónico en estos temas que ayude a asegurar el bienestar de los territorios de pueblos indígenas en aislamiento y contacto inicial y también atender las necesidades de adaptación y mitigación del cambio climático. Ya hace un tiempo la sociedad civil viene desarrollando experiencias de cooperación interinstitucional como lo evidencia la Red RAISG. El desafío del cambio climático y sus demandas de conocimiento acelerado e innovador sugieren invertir más en estas experiencias ampliando su cobertura hacia los ecosistemas ligados a la Amazonia como los Andes o el nordeste brasileño a fin de mejorar y ampliar la escala de la incidencia sobre paisajes y paisajes conexos.

A nivel de los gobiernos regionales se han venido dando iniciativas desde la sociedad civil como Madre de Dios Acre Pando MAP que luego fue integrando a los políticos a participar en varios de sus eventos. Como toda iniciativa dependió mucho de sus promotores y su accionar ha decaído luego de una década.

Recebido para publicação em 15 de outubro de 2015 Aceito em 21 de novembro de 2015

\section{REFERENCIAS}

BRYSK, A. Turning weakness into strength: the internationalization of Indian rights. Latin American Perspectives, v. 23, n. 89, 38-57. 1996.

BUNKER, S. Under developing the Amazon. Extraction, 
unequal exchange and the failure of the modern state. The University of Chicago Press. Chicago and London. 1985.

CIFOR, Redd. Forest governance and rural livelihoods. The emerging agenda. Springate-Baginski and Eva Wollenberg (eds.). 279p. 2010.

COICA. Alternativa de REDD+ Indígena. Territorios de vida plena para enfriar el planeta, Lima. 2011.

DANDLER, J.; URDAY, A.G.; LARRABURRE, J.L.; MOORE, T.; ORTEGA, R.R.; SIVERTSEN, L.; et al. Pueblos Indígenas de la Amazonia Peruana y Desarrollo Sostenible. Lima, Perú: Hivos, OIT, PNUD. 1998.

DJEUMO, A. The development of community forests in Cameroon: origins, current situation and constraints. RDFN paper 25b (i) - July. 2001.

GOULDING, M.; BARTHEM, R.; FERREIRA, E. J. G. The Smithsonian Atlas of the Amazon, Washington, DC: Smithsonian Books. 2003.

HABERMAS, J. Between facts and norms. Cambridge, UK: Polity Press. 1997.

HALL, A. (Ed.). Amazonia at the crossroads. The challenge of sustainable development. London: Institute of Latin American Studies. 2000.

HECHT, S.; COCKBURN, A. The fate of the forest. Developers, destroyers and defenders of the Amazon. New York: Harper. 1990

MORAN, E. F. Developing the Amazon. Bloomington: Indiana University Press. 1981.

MUMME, S. P.; KORZETZ, E. Democratization, politics, and environmental reform in Latin America. In G. J. MacDonald, G. J. Nielson, \& M. P. Stern (eds.). Latin American Environmental Policy in International Perspective. Boulder, CO: Westview Press. 1997.

OSTROM, E. Governing the commons: the evolution of Institutions for Collective Action. London. Cambridge University Press. 1990.

ROSALES, M. Comercio Internacional de Especies silvestres amenazadas y su influencia en el desarrollo sostenible. Tesis de Doctorado. Lima, Universidad Nacional Federico Villarreal. 2013.

SORIA, C. Ecuador's and Peru's environmental policies: oil, environment and indigenous peoples in the Amazon. South African Journal of Environmental Law and Policy, v. 11, n. 1, p. 81-125. 2004.
Amazonia, en Birx, James (Ed.). 21st Century Anthropology: a reference handbook. Los Angeles, Londres, New Delhi, Singapur y Washington, DC. Sage Publications. 2010.

Environmental law and civil society in Chile, Ecuador and Peru. Scholars Press. Saarbrucken. 2014.

The impact of cites cop 122002 decision on mahogany on Peru's Timber Trade', Cites, on a forthcoming book by Cites, Geneva. 2015a.

Los pueblos indígenas en aislamiento y contacto inicial de la Amazonia en el derecho internacional. Revista Estructura Salvaje, 2015 Edición Especial Amazonía, Lima, UNMSM. 2015b.

Increased relevance and influence of Free Prior Informed Consent (FPIC), REDD, and Green Economy principles on sustainable commons management in Peru, prepared for IASC 2015 Conference at the University of Alberta, Edmonton, May 25-29, Journal of Sustainable Development and Policy (JSDLP), of the Afe Babalola University, in Nigeria, in v. 5, n. 1, Spring (July). 2015c.

Los pueblos indígenas en aislamiento y contacto inicial y los bosques de la Amazonia. En: Infoandina, Lima, en http://www.infoandina.org/content/los-pueblosind\%C3\%ADgenas-en-aislamiento-y-contacto-inicial-ylos-bosques-de-la-amazon\%C3\%ADa, revisado 25 de abril de 2016. 2015d

Legislación Amazónica de Protección de Los Pueblos Indígenas. En: Actualidad Jurídica, Junio, Lima, Gaceta Jurídica. 2015e.

(en prensa) Consulta Previa y Gestión Ambiental en 6 lotes petroleros en Amazonia Peruana, preparado para el Coloquio Gobernanza local, pueblos indígenas e industrias extractivas en América Latina: transformaciones y continuidades, Agosto 11, a ser publicado en número especial del Boletín de la Sociedad Suiza de Americanistas BSSA.

VARESE, S. The new environmentalists movement of Latin American Indigenous People. In S. Brush \& D. Stabinsky (Eds.). Valuing local knowledge: indigenous people and intellectual property rights. Washington, DC: Island Press. p.122-142, 1996. 


\section{AMAZON IN THE FUTURE OF SOUTH AMERICA: identifying strategic socio- environmental axes for the South-South cooperation}

\author{
Carlos Antonio Martin Soria Dall’Orso
}

This article seeks to identify opportunities for the development of public policies and environmental projects that could support the transition to the Green Economy, from a proposal that meets the needs and opportunities that Amazon offers for a diverse development, with low carbon and social, environmental and economic sustainability. It reviews the current situation of the extractive model, which has limited development of the legal and philosophical apparatus of human rights, towards a model based on the understanding of the human rights paradigm, helping to build more egalitarian relationships, providing opportunities for the development of public policies that favor the diversification of the development model and the incorporation of low carbon projects, to support the sustainability of the Amazon and its people. The key legal and institutional issues affecting indigenous and rural populations are reviewed in order to strengthen the model of diverse development, relying on the development of the skills its population has.

Keywords: Amazon. Green economy. Sustainability. Carbon. Peoples.

\section{L'AMAZONIE DANS L'AVENIR DE L'AMÉRIQUE DU SUD: identification des axes stratégiques socio-environnementaux pour une coopération Sud-Sud}

Carlos Soria

Larticle cherche à identifier les opportunités de développement de politiques publiques et de projets socio-environnementaux qui sont à la base d'une transition vers une Économie Verte à partir d'une proposition qui prend en considération les besoins et les possibilités qu'offre l'Amazonie pour un développement diversifié, faible en émission de carbone et qui offre une durabilité sociale, environnementale et économique. Il examine la situation actuelle du modèle extractif, limité quant au développement de l'appareil juridique et philosophique concernant les droits de l'homme, pour aller vers un modèle qui, basé sur la compréhension du paradigme des droits humains, aide à établir des relations plus égalitaires et offre des opportunités pour le développement de politiques publiques favorisant la diversification du modèle de développement et l'intégration de projets faibles en émission de carbone pour soutenir le développement durable de l'Amazonie et de ses habitants. Les principaux problèmes légaux et institutionnels qui touchent les populations indigènes et rurales sont réexaminés afin de consolider le modèle d'un développement diversifié fondé sur les capacités de ses habitants.

Mot-ClÉs: Amazonie. Économie verte. Développement durable. Emission de Carbone. Populations.

Carlos Antonio Martin Soria Dall'Orso - Doutor em Filosofia em Estudos Latino-Americanos. Pesquisador e Consultor da Pontificia Universidade Católica do Perú, Universidad Nacional Agraria La Molina, Universidad San Ignacio de Loyola, Universidad Pedro Ruiz Gallo (INTE PUCP). Integra os Núcleos de pesquisa em Ética e Meio Ambiente, Florestas e Interculturalidade e Meio Ambiente.

Publicações recentes: Las obligaciones del estado peruano sobre la situación ambiental de la Amazonia, Revista virtual POLEMOS, Lima, PUCP. 2015; A propósito de Sociedad Civil y Derecho Ambiental en Chile, Ecuador y Perú. Revista UVA, Lima. 2015; Los pueblos indígenas en aislamiento y contacto inicial y los bosques de la Amazonia. Infoandina, Lima. 2015. 
